\title{
Acute and chronic desalivation and isotonic saline preference in rats
}

\author{
RODERICK WONG \\ University of British Columbia, Vancouver, Canada V6T IW5 \\ and \\ LEON KRAINTZ \\ Department of Oral Biology, Faculty of Dentistry, University of British Columbia \\ Vancouver, Canada V6T 1 W5
}

\begin{abstract}
The saline preference of acute and chronic desalivated rats was studied in two strains of rats. Desalivated and sham-operated rats were tested in the two-bottle situation where their consumption of $0.9 \% \mathrm{NaCl}$ and water was measured daily. Experiment 1 dealt with Long-Evans hooded rats which were tested for 20 consecutive days immediately after the operation. Experiment 2 dealth with BD IX agouti Norway rats which were tested for 10 consecutive days 140 days after the operation. The results of both experiments indicated that desalivated rats show greater saline preference than control rats during most of the initial 10 test sessions.
\end{abstract}

In an extensive monograph on the effects of desalivation on the rat's food and water intake, Vance (1965) concluded that the presence or absence of saliva is very important in regulating the amount of $\mathrm{NaCl}$ a rat will ingest. This conclusion was drawn from the results of an experiment comparing sodium preference among the desalivates. Vance observed an exaggerated $\mathrm{NaCl}$ preference among desalivated rats that was evident mainly during the immediate postoperative period. About 30 days after the operation, the group differences almost disappeared. Vance did not assess the statistical significance of his results, an omission due in part to the small $\mathrm{N}$ of his groups (six control and four desalivated animals). Only a single reading was taken over a 48 -h period of a number of $\mathrm{NaCl}$ solutions varying in concentration. We feel that daily readings of the animal's intake over a substantial period of time is preferable to the "one-shot" test.

We feel that it is necessary to validate Vance's (1965) results under conditions more amenable to statistical analysis. The present experiment was designed to compare the isotonic saline intake of desalivated and sham-operated rats over a number of days once the operation was completed. Aside from repeating the observations over a number of sessions, we also incorporated a number of other

This research was aided in part by a grant from the National Research Council of Canada, 67-0247, to R. Wong, and by one from the U.B.C. Research Committee to L. Kraintz. We thank Ken Shimizu for his help in collecting the data for Experiment 1 and Beverly Clark for her help in collecting the data for Experiment 2 and in analyzing the data of both experiments. procedural changes that made our design more sensitive than the one used by Vance. His method of comparing the volume of saline consumed by the groups can lead to errors due to individual variation in total fluid consumption within and between groups. A saline preference score expressed as percentage of fluid volume obviates this problem of variability.

We tested the animals with isotonic saline mainly because maximal differences between desalivates and controls occurred with this solution. With solutions of lower and higher tonicity, differences appear to be less obvious in Vance's rats. An inspection of the preference-aversion function of the two groups' saline intake suggests minimal differences when $0.5 \%$ and $2.0 \% \mathrm{NaCl}$ were the test solutions. A recent study in this laboratory has revealed that when desalivated and sham-operated rats were tested 80 days postoperatively, the desalivated rats ingested less hypertonic saline $(2.0 \%)$ than the controls (Wong \& Kraintz, 1977). This finding was interpreted in terms of the fact that normal rats lose a substaritial amount of saline in their saliva when they groom. Because desalivated rats do not groom, they conserve more sodium than the intact rats that can groom. For this reason, the desalivated animals showed a lower preference for the hypertonic saline than did the controls.

In the present experiment, we investigated the immediate effects of desalivation on the rat's consummatory behavior in the two-bottle situation. We compared the daily saline preference of desalivated and sham-operated rats for $0.9 \% \mathrm{NaCl}$ immediately after their operations over 20 consecutive sessions. 


\section{EXPERIMENT 1}

\section{Method}

The subjects were 14 male Long-Evans hooded rats ranging in weight from 260 to $300 \mathrm{~g}$ at the beginning of the experiment. The animals were randomly assigned to one of two conditions. The experimental group consisted of rats that were desalivated by having had their submaxillary and parotid ducts ligated. The operative procedure was carried out under Nembutal anesthesia. A midline incision was used to expose the ducts of the submaxillarysublingual and parotid glands. The ligation procedure was chosen because it avoids damage to the nearby ramus manibularis nerve. This sensory-motor branch of the fifth cranial nerve is more likely to be damaged had we used the extirpation procedure of desalivation. Ligation of the salivary ducts prevents or interrupts salivary flow from the salivary glands, and a comparison of the effects of duct ligation and saliverectomy on drinking patterns indicates identical results (Kissileff, 1969). In the present experiment, the control group consisted of animals that received a sham operation in the ventral surface of the neck. The wounds were closed by silk sutures.

The animals were housed in single stainless steel cages measuring $17.8 \times 24.2 \times 17.8 \mathrm{~cm}$ and were tested the day after their operations. Their daily consumption of water and $0.9 \% \mathrm{NaCl}$ solution was recorded throughout the experiment, and the leftright position of the water and saline was switched from day to day in order to control for any tendency of the animals to prefer a given position. Purina Lab Chow was placed on the floors of the cages and the animals had ad-lib access to this food. The fluid intake was measured for 20 days.

\section{Results and Discussion}

The results of the two-bottle test were first analyzed in terms of a preference score [(saline intake/total fluid intake) $\times 100$ ]. Statistical analysis of the data plotted in blocks of 2 days in Figure 1 indicates that the desalivated group showed greater saline preference than the sham-operated group, $\mathrm{F}(1,12)=8.80, \mathrm{p}<.025$. There was also a significant effect due to days, $F(19,247)=12.04, p<.001$, but the Groups by Days interaction was not statistically significant $(\mathrm{p}>.05)$.

An inspection of Figure 1 and a statistical analysis indicate that aside from group differences, the rats in both groups showed a decline in saline preference over successive test sessions. It may appear that this finding is at odds with the conception that the preference of isotonic saline over water is strong and persistent (Falk, 1961). However, notice that the data are plotted in blocks of two trials and it is not until the eighth day (Block 4) that the control rats are essentially taking no isotonic saline. It is not unusual for rats to show a decreasing tendency to ingest $\mathrm{NaCl}$ after successive exposures to this solution. Devenport (1973) reported that the rat's ingestion of saline decreases in quantity over a period of 2 or 3 days.

The data were also analyzed in terms of the total fluid (water + saline) consumption of the desalivated and control animals. An analysis of variance indicated that the desalivated rats drank more than the controls, $F(1,12)=6.43, p<.05$. Neither

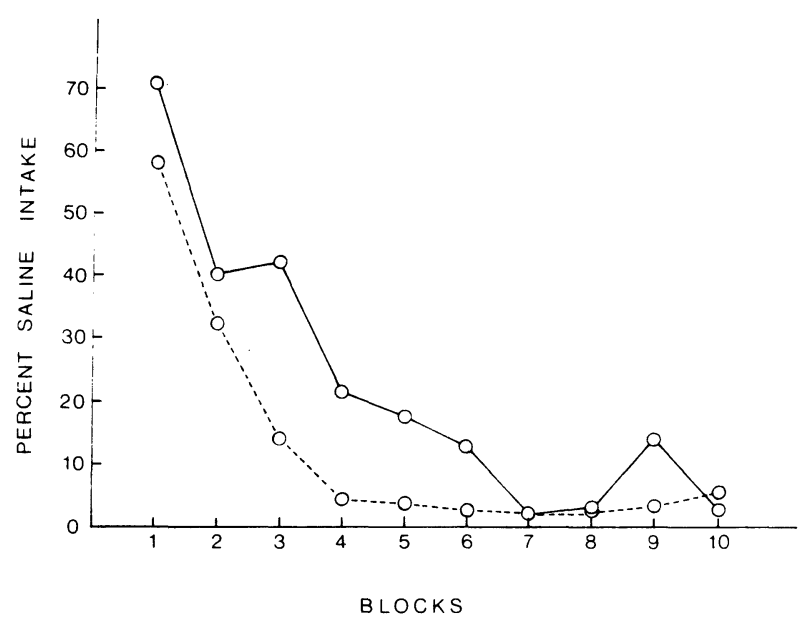

Figure 1. Saline preference [(saline/saline-water) $\times 100]$ of desalivated $\left(\mathrm{O}_{-} \mathrm{O}\right)$ and sham-operated $\left(\mathrm{O}-\mathrm{-}_{--\mathrm{O})}\right)$ hooded rats for $0.9 \% \mathrm{NaCl}$ over blocks of two sessions.

the days nor the Groups by Days interaction was significant $(\mathrm{p}<.05)$. Such results are consistent with Vance's (1965) finding of greater fluid intake among the desalivated rats.

\section{EXPERIMENT 2}

Although the finding that desalivated rats show greater saline preference than the controls validates Vance's (1965) previous observations to this effect, we also found that the group differences disappear after a number of test sessions. It is impossible to ascertain whether the disappearance of group differences was due to successive exposure to the twobottle situation or to the possibility that postoperative effects may be minimized after a certain period after the operation. Vance (1965) had found an absence of group differences in $0.9 \% \mathrm{NaCl}$ intake when the rats were retested 30 days after the operation. However, it should be noted that these rats had been exposed to an ascending series of $\mathrm{NaCl}$ solutions during their original tests. In order to ascertain whether the absence of group differences are due to continuous exposure to saline or due to time following the operation, it is necessary to conduct a similar experiment with operated rats that have not been exposed to saline solution for at least $\mathbf{3 0}$ days prior to the two-bottle test.

The present experiment was conducted with rats that had been operated 140 days before the present test sessions. These rats had previously been tested in an experiment where their intake of $2.0 \% \mathrm{NaCl}$ and water had been assessed (Wong \& Kraintz, 1977). The results of that experiment indicated that desalivated rats ingest less hypertonic saline solution than the controls. Because of this apparent anomaly, it is highly important to study the same animals in 
the two-bottle situation where their intake of isotonic saline and water would be measured. If the results of this experiment indicate higher isotonic saline preference among desalivates, then Vance's original findings would receive additional confirmation. In addition, such results would suggest that the interval between operation and testing alone does not account for the disappearance of group differences in saline preference.

\section{Method}

The subjects were 13 male Norway rats of the BD IX strain ranging in weight from 400 to $500 \mathrm{~g}$. These animals were among the 16 rats used in an earlier experiment (Wong \& Kraintz, 1977). In the 60 days intervening the end of the previous experiment and the beginning of the present one, two of the sham-operated and one of the desalivated rats had died. Thus, we were left with seven desalivated and six sham-operated rats in the present experiment. The operation procedure was as described in Experiment 1 , except that wound clips were used to close the incisions of the rats in the present experiment.

The housing and two-bottle testing conditions were similar to those employed in Experiment 1. In the present experiment, the rats were tested for 10 consecutive days.

\section{Results and Discussion}

The data were first analyzed in terms of the saline preference ratio described in Experiment 1. The results are plotted in Figure 2, and it can be seen that the desalivated group showed a higher saline preference than controls during most of the 10 test sessions. This difference was statistically significant, $F(1,11)$ $=6.37, \mathrm{p}<.05$. Unlike the results of Experiment 1 , there was no significant change in saline preference of the rats over the consecutive test sessions $(\mathrm{p}>.05)$.

The analysis of the total fluid (water + saline) consumption of the desalivated and control animals indicated that the experimental rats drank more, $F(1,11)=5.77, p<.05$. There were no significant differences due to the days or the Groups by Days interaction $(p>.05)$. All of these results are consistent with those obtained in Experiment 1.

In general, the results of Experiment 2 substantiate the finding that desalivated rats show a higher preference for isotonic saline than the sham-operated rats. In this respect, there is agreement between the findings by Vance (1965) and those from this laboratory. When considering the fact that the rats in Vance's experiment were albino, the ones in Experiment 1 were hooded, and those in Experiment 2 were agouti, it appears that Vance's original contention that desalivated rats show greater saline preference than control animals has substantial support.

It is interesting that although, in a previous experiment, the rats of Experiment 2 showed a lower preference for hypertonic saline (Wong \& Kraintz, 1977), they nevertheless exhibited a greater preference for isotonic saline in the present experiment.

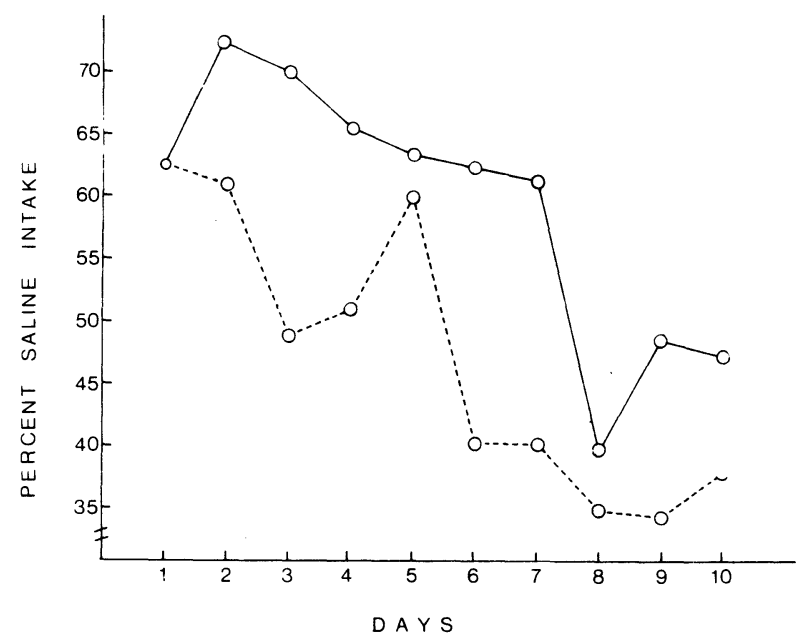

Figure 2. Saline preference [saline/saline-water) $\times 100$ ] of desalivated $\left(\mathrm{O}_{-} \mathrm{O}\right)$ and sham-operated $\left(\mathrm{O}-\mathrm{-O}^{-}\right)$agouti rats over days.

Water was the only fluid offered to these animals during the 60 days between the end of the experiment involving hypertonic saline and the beginning of the present one with isotonic saline. It should also be noted that the present experiment was conducted 140 days after the rats were operated on. Apparently, desalivation effects are long-lasting. This result suggests that the reduction of group differences over consecutive test sessions observed in Experiment 1 was not due to the time between operation and testing. Instead, we believe that the animal's reduction in saline intake was due to their continuous exposure to saline during the test sessions. Although the agouti rats in Experiment 2 did not show as rapid a decrease in saline intake as the hooded rats in Experiment 1, they seem to show a tendency in this direction. Because of the large within-group variability, the agouti rats' decline in saline preference was not statistically significant $(p>.05)$ when an analysis of variance was performed on the data. However, a comparison between the rats' mean saline preference ratio score during the first and last three test sessions with a related samples nonparametric test (Walsh, 1949) indicated a decrease significant at the .05 level. Genetic differences may be responsible for the differences in rate of decrease of agouti and hooded rats.

\section{REFERENCES}

Devenport, L. D. Aversion to a palatable saline solution in rats: Interactions of physiology and experience. Journal of Comparative and Physiological Psychology, 1973, 83, 98-105.

FALK, J. L. The behavioral regulation of water-electrolyte balance. In M. R. Jones (Ed.), Nebraska symposium on motivation. Lincoln: University of Nebraska Press, 1961.

KISSILEFF, H. R. Food-associated drinking in the rat. Journal of 
Comparative and Physiological Psychology, 1969, 67, 284-300.

VANCE. W. B. Observations on the role of salivary secretions in the regulation of food and fluid intake in the white rat. Psychological Monographs, 1965, 79(5, Whole No. 598).

WALSH. J. E. Applications of some significance tests for the median which are valid under very general conditions. Journal of
American Statistical Association, 1949, 44, 342-355.

Wong, R., \& KRAINTz, L. Desalivation and saline ingestion in rats. Behavioral Biology, 1977, 19, 130-134.

(Received for publication November 22, 1976; accepted January 23.1977.) 